\title{
Some Dilute Solution Properties of Highly Isotactic Polyacrylonitrile in Aq. Nitric Acid
}

\author{
Hitoshi Yamazaki, Yukio MiYazaki, Masatoshi SaIto, \\ and Kenji KAMIDE* \\ Fundamental Research Laboratory of Fibers and Fiber-Forming Polymers, \\ Asahi Chemical Industry Co., Ltd., 11-7 Hacchonawate, \\ Takatsuki, Osaka 569, Japan
}

(Received August 2, 1990)

\begin{abstract}
An attempt was made to investigate the dilute solution properties of highly isotactic polyacrylonitrile (PAN) dissolved in aq. nitric acid and to estimate the molecular parameters, such as the unperturbed chain dimension $A$ and the characteristic ratio $C_{\infty}$. For this purpose a highly isotactic polymer with isotactic triad content of $73.4 \%$ was synthesized by irradiating $\gamma$-ray on acrylontrile-urea canal complex at $-78^{\circ} \mathrm{C}$ and was subjected to successive precipitation fractionation, using dimethyl sulfoxide as solvent and toluene as precipitant, into 7 fractions. Among them 2 fractions with isotactic triad of 71.0 and $77.9 \%$, respectively were employed for further study. The weight-average molecular weight $M_{w}$ and the second virial coefficient $A_{2}$ of these fractions in $70 \mathrm{wt} \%$ aq. nitric acid soln. were measured by the light scattering method. The $70 \mathrm{wt} \%$ aq. nitric acid soln. was predicted to be able to dissolve perfectly-isotactic PAN with $M_{w}$ of $1.5-2.6 \times 10^{5}$ at room temperature. The Mark-Houwink-Sakurada equations in aq. nitric acid solutions differ significantly depending on the stereoregularity of PAN employed, even if highly concentrated acids are used. A $57.5 \mathrm{wt} \%$ aq. nitric acid soln. is determined, by the dependence of $A_{2}$ on the nitric acid concentration, to be a Flory's theta solvent for PAN with isotactic triad of $71.0 \%$ at $25^{\circ} \mathrm{C}$. A of PAN increases rapidly with isotactic triad content, especially in the range higher than $50 \% . C_{\infty}$ of PAN is always far larger than the calculated value of non-polar Monte Calro chain for polypropyrene [P. J. Flory et al., J. Am. Chem. Soc., 88, 639 (1966)] over the whole range higher than $50 \%$ isotactic diad.
\end{abstract}

KEY WORDS Dilute Solution Properties / Isotactic Polyacrylonitrile / Nitric

Acid / Light Scattering / Unperturbed Chain Dimension /

It is widely wellknown that the solubility of vinyl-type polymer against solvents decreases notably with increasing stereoregularity of the polymer $^{1}$ and that the Mark-Houwink-Sakurada (MHS) equation, relating the limiting viscosity number $[\eta]$ and the molecular weight $M$ and the relation between the second-virial coefficient $A_{2}$ and $M$, and the unperturbed chain dimension $A$ are sometimes significantly dependent on the stereoregularity of the polymers, especially when the solvents employed are rather poor. ${ }^{1,2}$ In a previous paper, ${ }^{2}$ we disclosed on polyacrylonitrile (PAN) with isotactic triad ( $\mathrm{mm}, \mathrm{m}$ denotes meso configuration) content of $52 \%$, prepared by $\gamma$-ray irradiation on acrylonitrile(AN)-urea canal complex at $-78^{\circ} \mathrm{C}$ (hereafter, referred to as $\gamma$-PAN-1) that (1) $\gamma$-PAN-1 never dissolves even at $80^{\circ} \mathrm{C}$ into $N, N$-dimethylformamide (DMF) (i.e., the solubility is zero), (2) $A_{2}$ of $\gamma$ PAN-1 in dimethyl sulfoxide (DMSO) at $25^{\circ} \mathrm{C}$ is almost half of that for PAN with $\mathrm{mm}$ content of $26 \%$, which was prepared in aq. solution by redox catalyst (referred to as R-PAN), ${ }^{3}$ (3) the

* To whom all correspondence should be addressed. 
parameters in the MHS equation evaluated for $\gamma$-PAN-1 in DMSO at $25^{\circ} \mathrm{C}$ slightly differed from those for R-PAN in the same solvent, (4) $A$ for $\gamma$-PAN-1 in DMSO was $7 \%$ larger than that for R-PAN, (5) there is small, but significant dependence of the MHS parameters in aq. nitric acid on the stereoregularity of the polymers when the polymers with $\mathrm{mm}$ content $26 \%$ and $52 \%$ are compared. Now, it is highly expected that if the polymers with much higher stereoregularity are available, the effect of the isotacticity of PAN on its dilute solution properties becomes more explicit, and the characteristic nature of aq. nitric acid toward PAN can be more clearly understood.

\section{EXPERIMENTAL}

\section{Polymer}

Reagent grade urea was purified by recrystallization method from water-methanol mixture and was mixed with acrylontrile (AN, purity $99.5 \mathrm{wt} \%$ or more), to which $n$-butyl mercaptan as chain transfer reagent was added in advance to make a $1 \mathrm{~mol} \%$ solution, to give two kinds of mixtures of $\mathrm{AN}$ and urea ( $1: 3$, and $1: 1.5$ by mol ratio). By setting down these mixtures for 7 days at $-78^{\circ} \mathrm{C}$, the AN-urea canal complex was formed completely. Keeping the complex at $-78^{\circ} \mathrm{C}$ and by irradiating with $\gamma$-ray from Cobalt 60 $(14000 \mathrm{Ci})$ at total dose of $9 \times 10^{4}$ and $15 \times$ $10^{5}$ roentgen $(\mathrm{R}), \mathrm{AN}$ in canal complex polymerized to PAN, which was washed repeatedly with water and methanol to remove urea and unreacted $\mathrm{AN}$ and was dried in vacuo at room temperature. The polymers thus prepared (sample code $\gamma$-PAN-2 and $\gamma$-PAN-3) have $[\eta]$ in $70 \mathrm{wt} \%$ aq. nitric acid soln. at $25^{\circ} \mathrm{C}$ of 283 and $214 \mathrm{~cm}^{3} \mathrm{~g}^{-1}$ and $\mathrm{mm}$ content of 73.4 and $54.2 \%$ as determined by ${ }^{13} \mathrm{C}$ NMR method, ${ }^{4}$ respectively. In addition to these polymers $(\gamma$-PAN), a fraction (R-PAN-4, $\mathrm{mm}$ content $26.0 \%$ ) of R-PAN, prepared in the previous paper $^{3}$ and a fraction $(\gamma-1-4, \mathrm{~mm}$ content $53.2 \%)^{2}$ separated from sample $\gamma$-PAN-1 were also used in this study.

\section{${ }^{13} C N M R$}

${ }^{13} \mathrm{C}$ NMR measurements on $\gamma$-PAN were made on FT-NMR JEOL FX200 at $27^{\circ} \mathrm{C}$ using the mixture of $65 \%$ nitric acid- $d_{1}$ in $\mathrm{D}_{2} \mathrm{O}$ and $70 \%$ nitric acid in $\mathrm{H}_{2} \mathrm{O}(2: 1$, volume ratio) as a solvent and dioxane as an internal standard. In order to suppress the Nuclear Overhauser Effect (NOE), inverse gated decoupling method $^{5}$ was employed. Triad tacticities (contents of $\mathrm{mm}, \mathrm{mr}$, and $r r$ sequences; $r$, racemo configuration) of the unfractionated and fractionated $\gamma$-PAN were evaluated from the area of methine and cyano carbon peaks of these polymers. ${ }^{4}$

\section{Fractionation}

The samples $\gamma$-PAN-2 and $\gamma$-PAN-3 were fractionated into 7 and 6 fractions, respectively by successive precipitation fractionation (SPF) method using DMSO as solvent and toluene as precipitant. SPF was operated under the following conditions: Starting polymer concentration $c\left(\mathrm{~g} \mathrm{~cm}^{-3}\right), 0.0125$; initial concentration, 0.007 at the first fractionation step; temperature, $30^{\circ} \mathrm{C}$. The 2 nd (denoted as sample code $\gamma-2-2)$ and 3rd $(\gamma-2-3)$ fractions of the sample $\gamma$-PAN-2 and the 3rd fraction ( $\gamma-3-3)$ of the sample $\gamma$-PAN-3 were subjected to the further study.

\section{Light Scattering}

For light scattering measurements five kinds of solvents, DMSO and aqueous nitric acid with the concentrations of $60,63,66$, and $70 \mathrm{wt} \%$ were used. In order to avoid degradation of polymer in aq. nitric acids, the polymer solutions with the concentration of $0.001-0.005 \mathrm{~g} \mathrm{~cm}^{-3}$ were individually prepared at $25^{\circ} \mathrm{C}$ and then immediately filtrated through a membrane, Floropore ${ }^{\mathbf{R}}$ with pore diameter of $0.45 \mu \mathrm{m}$, manufactured by Sumitomo Denko Co. (Osaka). Due to lack of a commercially available semi-permeable membrane durable to the concentrated nitric acid 
the PAN/aq. nitric acid soln. systems were employed without any dialysis for the light scattering measurements. When DMSO was used as solvent, a mother solution prepared at $80^{\circ} \mathrm{C}$ was centrifuged at $20^{\circ} \mathrm{C}$ for $70 \mathrm{~min}$ under the gravity of $5 \times 10^{4} \mathrm{~g}$, followed by dilution with DMSO and filtration through the above-mentioned membrane.

Refractive index increments (RII) of the systems including $\gamma$-PAN-3/60-70\% aq. nitric acid solution $\left[(\partial n / \partial c)_{\phi N} ; \phi_{N}\right.$, volume concentration of nitric acid], $60-70 \%$ aqueous nitric acid solution $\left[\left(\partial n_{0} / \partial \phi_{N}\right), n_{0}\right.$, refractive index of aq. nitric acid], and $\gamma$-PAN-3/DMSO $\left[(\partial n / \partial c)_{\mathrm{D}}\right]$, were determined at $25^{\circ} \mathrm{C}$ using a Shimadzu differential refractometer type DR-4 with incident light wave length $\lambda_{0}=633 \mathrm{~nm}$. $(\partial n / \partial c)_{\phi N}$ and $\left(\partial n_{0} / \partial \phi_{N}\right)$ were found almost independent of the concentration of nitric acid respectively at $25^{\circ} \mathrm{C}$ and to be 0.0967 and $0.131 \mathrm{~cm}^{3} \mathrm{~g}^{-1}$. $(\partial \mathrm{n} / \partial c)_{\mathrm{D}}$ was $0.038 \mathrm{~cm}^{3} \mathrm{~g}^{-1}$ at $25^{\circ} \mathrm{C}$. When we analyzed the light scattering data on the fractionated PAN with triad tacticity ranging from 26 to $77.9 \%$ in aq. nitric acid solution, $0.0967 \mathrm{~cm}^{3} \mathrm{~g}^{-1}$ was used as the RII value of these systems, providing that RII is virtually unchanged over wide variations of the molecular weight and the tacticity.

The light scattering measurements were made at $25^{\circ} \mathrm{C}$ over the scattering angle ranging from 30 to $150^{\circ}$ (step 10 degree) on a light scattering apparatus DLS 700, manufactured by Otsuka Electronics (Osaka) with a vertically polarized incident beam $\left(\lambda_{0}=633 \mathrm{~nm}\right)$. The scattered light intensity was calibrated in advance with literature value $\left(1.184 \times 10^{-6}\right.$ $\left.\mathrm{cm}^{-1}\right)^{6}$ of reduced Rayleigh ratio of benzene.
The data were analyzed according to the Zimm's procedure.

\section{Limiting Viscosity Number}

The solution viscosity was measured in a modified Ubbelohde suspension type viscometer at the temperature of $25 \pm 0.01^{\circ} \mathrm{C}$. [ $\left.\eta\right]$ was determined from a Huggins plot.

\section{RESULTS AND DISCUSSION}

Figure $1 \mathrm{a}$ and $\mathrm{b}$ show ${ }^{13} \mathrm{C}$ NMR spectra of the cyano carbon of two fractions $\gamma-2-2$ (a) and $\gamma-2-3$ (b) in $65 \%$ nitric acid- $d_{1} / \mathrm{D}_{2} \mathrm{O}$ system. Only three main peaks were observed for each samples, while if we use the deuterated DMSO as a solvent, the peaks corresponding to pentad or higher order of regularity appeared. ${ }^{7} \mathrm{We}$ estimated the triad-tacticity (i.e., the molar fractions of iso $(\mathrm{mm})$, hetero $(\mathrm{mr})$, and syndio (rr) sequences) of these fractions from ${ }^{13} \mathrm{C}$ NMR spectra of the cyano carbon assuming that all of three peaks in the figures are attributed to three kinds of triad sequence:
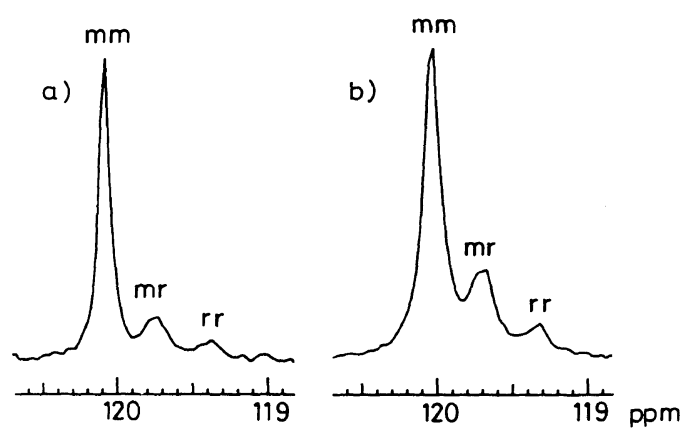

Figure 1. ${ }^{13} \mathrm{C}$ NMR spectra of cyano carbon of fraction $\gamma-2-2(a)$ and $\gamma-2-3(b)$ in $6.5 \%$ nitric acid- $d_{1} / \mathrm{D}_{2} \mathrm{O}$ system.

Table I. Triad tacticities of $\gamma$-PAN fractions determined by ${ }^{13} \mathrm{C}$ NMR method

\begin{tabular}{|c|c|c|c|c|c|c|c|c|c|}
\hline \multirow{2}{*}{$\begin{array}{l}\text { Sample } \\
\text { code }\end{array}$} & \multicolumn{3}{|c|}{$\mathrm{CN}^{\mathrm{a}}$} & \multicolumn{3}{|c|}{$\mathrm{CH}^{\mathbf{b}}$} & \multicolumn{3}{|c|}{$A v^{c}$} \\
\hline & $m m / \%$ & $m r / \%$ & $r r / \%$ & $\mathrm{~mm} / \%$ & $m r / \%$ & $r r / \%$ & $m m / \%$ & $m r / \%$ & $r r / \%$ \\
\hline$\gamma-2-2$ & 78.1 & 16.5 & 5.4 & 77.7 & 18.5 & 3.8 & 77.9 & 17.5 & 4.6 \\
\hline$\gamma-2-3$ & 71.6 & 21.3 & 7.0 & 70.4 & 21.9 & 7.6 & 71.0 & 21.6 & 7.3 \\
\hline
\end{tabular}

a Cyano carbon. ${ }^{\mathrm{b}}$ Methine carbon. ${ }^{\mathrm{c}}$ Average value. 
From higher magnetic field, $m m, m r$, and $r r$ sequences. The results are summarized in Table I. Similar molar fractions of $\mathrm{mm}, \mathrm{mr}$, and $r r$ sequences, evaluated from the area of the peaks corresponding to methine carbon and to cyano carbon were obtained. The average triad tacticity values evaluated by the above two methods for two $\gamma$-PAN fractions were also compiled in the table. It was observed in the previous paper ${ }^{2}$ that $\mathrm{mm}$ contents of the fractions obtained by SPF method from $\gamma$-PAN-1 ( $\mathrm{mm}$ content, 52\%) were almost the same as that of the whole polymer. For highly isotactic PAN the situation is somewhat different; the average $\mathrm{mm}$ content of the fractions of $\gamma$-PAN-2 remarkably differs, approximately $5 \%$, from that of the whole polymer. This result strongly suggests that $\gamma$-PAN samples are mixtures of PAN molecules with different stereoregularity and in the SPF process $\gamma$-PAN with triad isotacticity higher than $70 \%$ is inherently fractionated not only by the molecular weight but also by the stereo regularity, if DMSO and toluene are used as solvent and precipitant, respectively, probably due to a noticeable decrease of the solubility as an increase of the tacticity.

Figure 2 depicts as an example the Zimm plot of sample code $\gamma-2-3 /$ DMSO system. The plot shows appreciable distortion particularly in lower scattering angle region of the poly- mer solution with higher concentration. The molecular parameters, such as $M_{w}, A_{2}$, and the $z$-average radius of gyration $\left\langle S^{2}\right\rangle_{z}^{1 / 2}$, all evaluated from the plots, are listed in Table II, in which the previous results ${ }^{2,3}$ on the R-PAN (R-PAN-4) and the sample code $\gamma-1-4$ with relatively low tacticity are also included. As will be described later, $M_{w}$ of both $\gamma-2-2$ and $\gamma-2-3$, evaluated in DMSO, are about 10 times larger than those evaluated in $70 \%$ nitric acid. These experimental facts imply that $\gamma$-PAN chains with higher isotacticity than $70 \%$ do not dissolve molecularly in DMSO at $25^{\circ} \mathrm{C}$, but tend to associate together, although the solutions with polymer concentration ranging from 0.001 to $0.005 \mathrm{~g} \mathrm{~cm}^{-3}$ look transparent just after these solutions were prepared. In fact both solutions of the sample code $\gamma-2-2$ and $\gamma-2-3$ occurred flocculation in 10 days at room

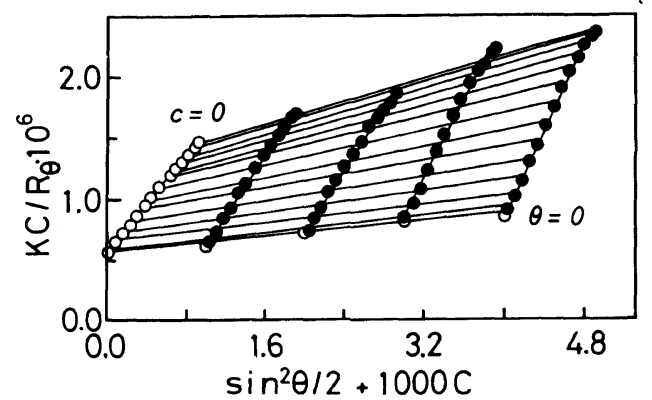

Figure 2. A Zimm plot for fraction $\gamma-2-3$ (b) in DMSO at $25^{\circ} \mathrm{C}$.

Table II. Results of light scattering and viscosity measurements on PAN-DMSO systems at $25^{\circ} \mathrm{C}$

\begin{tabular}{|c|c|c|c|c|c|c|}
\hline \multirow{2}{*}{$\begin{array}{l}\text { Sample } \\
\text { code }\end{array}$} & $m m^{\mathrm{a}}$ & \multirow{2}{*}{$M_{w} \times 10^{-4 \mathrm{~b}}$} & \multirow{2}{*}{$\frac{A_{2} \times 10^{3 \mathrm{c}}}{\mathrm{cm}^{3} \mathrm{~mol} \mathrm{~g}^{-2}}$} & \multirow{2}{*}{$\frac{\left\langle S^{2}\right\rangle_{z}^{1 / 2} \times 10^{8 \mathrm{~d}}}{\mathrm{~cm}}$} & \multirow{2}{*}{$\frac{[\eta] \times 10^{-2 \mathrm{e}}}{\mathrm{cm}^{3} \mathrm{~g}^{-1}}$} & \multirow{2}{*}{ Ref. } \\
\hline & $\%$ & & & & & \\
\hline$\gamma-2-2$ & 77.9 & 262.0 & -0.02 & 808 & 292 & This work \\
\hline$\gamma-2-3$ & 71.0 & 183.0 & 0.04 & 791 & 262 & This work \\
\hline$\gamma-1-4$ & 53.2 & 26.3 & 0.60 & 317 & 275 & Ref 2 \\
\hline R-PAN-4 & 26.0 & 15.5 & 1.53 & 230 & 190 & This work \\
\hline
\end{tabular}

a Content of meso-meso triad.

b Weight-average molecular weight.

c Second virial coefficient.

d $z$-Average radius of gyration.

e Limiting viscosity number. 
Table III. Results of light scattering measurements on PAN fractions in $70 \mathrm{wt} \%$ aq. nitric acid soln. at $25^{\circ} \mathrm{C}$

\begin{tabular}{|c|c|c|c|c|c|c|}
\hline \multirow{2}{*}{$\begin{array}{l}\text { Sample } \\
\text { code }\end{array}$} & \multirow{2}{*}{$\frac{\mathrm{mm}^{\mathrm{a}}}{\%}$} & \multirow{2}{*}{$M_{w}^{*} \times 10^{-4 \mathrm{~b}}$} & \multirow{2}{*}{$\frac{A_{2}^{*} \times 10^{3 \mathrm{c}}}{\mathrm{cm}^{3} \mathrm{~mol} \mathrm{~g}^{-2}}$} & \multirow{2}{*}{$\frac{\left\langle S^{2}\right\rangle_{z}^{1 / 2} \times 10^{8 \mathrm{~d}}}{\mathrm{~cm}}$} & \multirow{2}{*}{$M_{w} \times 10^{-4 \mathrm{e}}$} & \multirow{2}{*}{$\frac{A_{2} \times 10^{3 \mathrm{~h}}}{\mathrm{~cm}^{3} \mathrm{molg}^{-2}}$} \\
\hline & & & & & & \\
\hline$\gamma-2-2$ & 77.9 & 41.1 & 1.55 & 448 & 25.2 & 2.52 \\
\hline$\gamma-2-3$ & 71.0 & 28.4 & 1.56 & 423 & 17.4 & 2.54 \\
\hline$\gamma-3-3$ & 55.9 & 27.7 & 1.88 & 400 & 17.0 & 3.09 \\
\hline$\gamma-1-4$ & 53.2 & 43.2 & 2.05 & 393 & $26.3^{f}$ & 3.34 \\
\hline R-PAN-4 & 26.0 & 25.5 & 2.56 & 351 & $15.8^{\mathrm{g}}$ & 4.20 \\
\hline
\end{tabular}

a Content of meso-meso triad.

b The apparent weight-average molecular weight.

c The apparent second virial coefficient.

d The $z$-average radius of gyration.

e The weight-average molecular weight.

f The value determined using DMSO as solvent (ref 2).

g The value determined using DMF as solvent (ref 3).

h The second virial coefficient.

Table IV. Results of light scattering measurements on a $\gamma$-PAN fraction $(\gamma-2-3)$ in aq. nitric acid solutions with concentrations of $60,63,66,70 \mathrm{wt} \%$

\begin{tabular}{|c|c|c|c|c|c|}
\hline \multirow{2}{*}{$\begin{array}{c}\text { Solvent } \\
\mathrm{HNO}_{3} \text { conc. }\end{array}$} & \multirow{2}{*}{$M_{w}^{*} \times 10^{-4 a}$} & $A_{2}^{*} \times 10^{3 \mathrm{~b}}$ & $\left\langle S^{2}\right\rangle_{z}^{1 / 2} \times 10^{8 c}$ & \multirow{2}{*}{$M_{w} \times 10^{-4 d}$} & \multirow{2}{*}{$\frac{A_{2} \times 10^{3 \mathrm{e}}}{\mathrm{cm}^{3} \mathrm{~mol} \mathrm{~g}^{-2}}$} \\
\hline & & $\mathrm{cm}^{3} \mathrm{~mol} \mathrm{~g}^{-2}$ & $\mathrm{~cm}$ & & \\
\hline 60 & 23.7 & 0.45 & 392 & 15.0 & 0.73 \\
\hline 63 & 26.7 & 0.78 & 398 & 16.4 & 1.27 \\
\hline 66 & 25.6 & 1.29 & 410 & 15.7 & 2.10 \\
\hline 70 & 28.4 & 1.56 & 423 & 17.4 & 2.54 \\
\hline
\end{tabular}

a The apparent weight-average molecular weight.

b The apparent second virial coefficient.

c The $z$-average radius of gyration.

d The weight-average molecular weight.

e The second virial coefficient.

temperature. Judging from the values of $A_{2}$, DMSO is relatively poor and is very close to $\Theta$ solvent.

Figure 3 shows the Zimm plot of sample code $\gamma-2-3 / 70 \%$ aq. nitric acid system, which was prepared without dialysis. Contrary to the case when DMSO is used, the Zimm plot in the figure forms essentially perfect diamond shape. Apparent $M_{w}$, designated as $M_{w}^{*}$, apparent $A_{2}, A_{2}^{*}$, and $\left\langle S^{2}\right\rangle_{z}^{1 / 2}$ estimated for various $\gamma$-PAN and R-PAN fractions/aq. nitric acid soln. systems at $25^{\circ} \mathrm{C}$ and for the systems of sample code $\gamma-2-3$ in four aq. nitric acid solutions with nitric acid concentration of $60-70 \%$ at $25^{\circ} \mathrm{C}$ are listed in Tables III and IV, respectively.

$M_{w}^{*}$ and $M_{w}$ theoretically relate to the reduced scattering intensity $R(\theta)$ through the following equations, respectively ${ }^{8}$ :

$$
\begin{aligned}
& {\left[K^{*} c / R(\theta)\right]_{c \rightarrow 0}=1 /\left(M_{w}^{*} P(\theta)\right)} \\
& {[K c / R(\theta)]_{c \rightarrow 0}=1 /\left(M_{w} P(\theta)\right)}
\end{aligned}
$$

where $P(\theta)$ is a scattering function. $\theta$, the scattering angle. $K^{*}$ and $K$ are the optical constants of the polymer-binary solvents and polymer-single solvent systems, respectively, as 


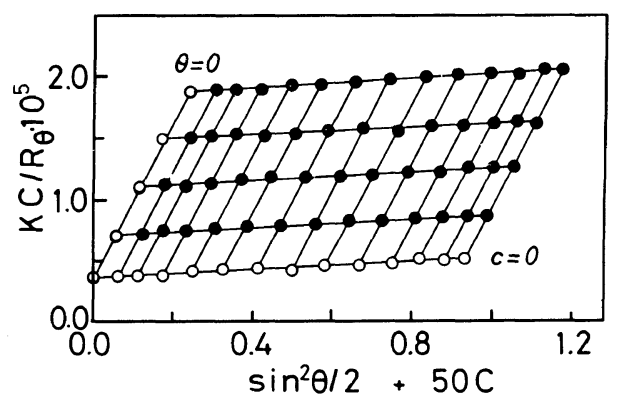

Figure 3. A Zimm plot for fraction $\gamma-2-3$ (b) in $70 \%$ aq. nitric acid soln. at $25^{\circ} \mathrm{C}$.

given by, ${ }^{8}$

$$
\begin{aligned}
& K^{*}=\left(2 \pi^{2} n_{0}^{2} / \lambda_{0}^{4} N_{\mathrm{A}}\right)(\partial n / \partial c)_{\phi N}^{2} \\
& K=K^{*}\left\{1+\xi_{\mathrm{a}}\left(\partial n_{0} / \partial \phi_{N}\right) /(\partial n / \partial c)_{\phi N}\right\}^{2}
\end{aligned}
$$

Here, $N_{\mathrm{A}}$ is Avogadro number; $\xi_{\mathrm{a}}$, the coefficient of the selective adsorption.

From eq 1, 1', 2, and $2^{\prime}, \xi_{\mathrm{a}}$ can be written as,

$$
\xi_{\mathrm{a}}=\left[\left(M_{w}^{*} / M_{w}\right)^{1 / 2}-1\right](\partial n / \partial c)_{\phi N} /\left(\partial n_{0} / \partial \phi_{N}\right)
$$

$\xi_{\mathrm{a}}$ values obtained for the samples R-PAN-4/ and $\gamma-1-4 / 70 \%$ aq. nitric acid systems approximately coincide with each other (averaged to be 0.203 ), irrespective of wide variation of the tacticity of the samples used. Note that for the above PAN samples true $M_{w}$ can be unconditionally determined by the light scattering measurements on DMSO solutions.

Putting the experimental values of $M_{w}^{*}$, $(\partial n / \partial c)_{\phi N}$, and $\left(\partial n_{0} / \partial \phi_{N}\right)$ for the samples $\gamma-2-2$ and $\gamma-2-3$, and average $\xi_{\mathrm{a}}(0.203)$ into eq 3 , we estimated $M_{w}$ of these samples.

$A_{2}$ can also be evaluated from $A_{2}^{*}$ through use of the relation ${ }^{9}$ :

$$
A_{2}=\left(M_{w}^{*} / M_{w}\right) A_{2}^{*}
$$

The values of $M_{w}$ and $A_{2}$ estimated thus for highly isotactic PAN fractions are also summarized in Tables III and IV. As shown in Figure 4, the molecular weight dependence of $A_{2}$ of the PAN with the triad isotacticity ranging from 26 to $78 \%$ in $70 \%$ aq. nitric acid solution is practically almost insignificant at least in the molecular weight range investi-

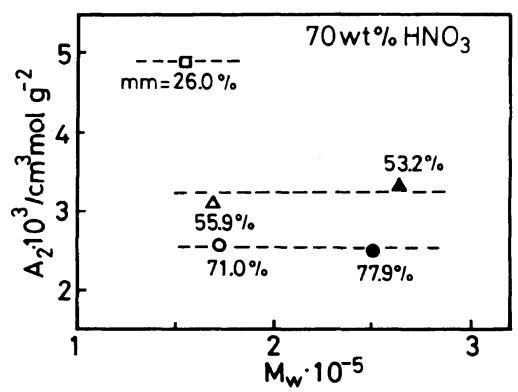

Figure 4. Plots of the second virial coefficient $A_{2}$ of PAN with $\mathrm{mm}$ (triad isotacticity) content ranging from 26 to $77.8 \%$ in aq. nitric acid solution versus the weight-average molecular weight $M_{w}$. $\bigcirc$, sample code $\gamma-2-3 ; 0, \gamma-2-2, \triangle$, $\gamma-3-3 ; \boldsymbol{\Delta}, \gamma-1-4 ; \square$, R-PAN-4. Numbers attached to the mark denote $\mathrm{mm}$ content $(\%)$.

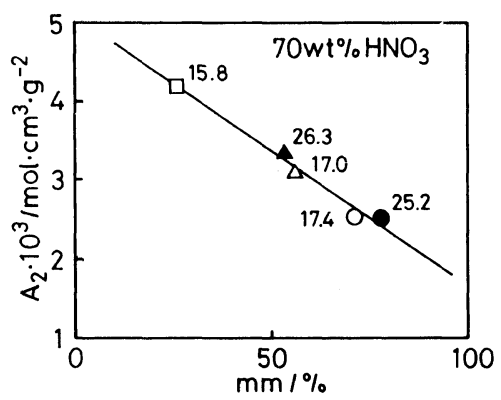

Figure 5. Effect of $m m$ (triad isotacticity) content on the second virial coefficient $A_{2}$ of PAN in $70 \%$ aq. nitric acid soln. Symbols have the same meanings as those in Figure 4. Numbers attached on the mark mean the weight-average molecular weight $M_{w} \times 10^{-4}$.

gated, if compared at the similar isotacticity level.

Figure 5 demonstrates the triad isotacticity dependence of $A_{2}$ of PAN in $70 \%$ aq. nitric acid at $25^{\circ} \mathrm{C} . A_{2}$ decreases monotonically as isotacticity increases. The relationship between $\mathrm{A}_{2}$ and triad tacticity $\mathrm{mm}$ of PAN can be approximately expressed by the following equation:

$$
\begin{array}{r}
A_{2}=5.07 \times 10^{-3}-3.40 \times 10^{-5} \mathrm{~mm} \\
\left(\mathrm{~mol} \mathrm{~cm} \mathrm{~g} \mathrm{~g}^{3}\right)
\end{array}
$$

The positive magnitude of $A_{2}\left(1.67 \times 10^{-3} \mathrm{~mol}\right.$ $\mathrm{cm}^{3} \mathrm{~g}^{-2}$ ) extrapolated from the experimental $A_{2}$ vs. mm content relations in Figure 5 to $100 \%$ isotacticity indicates that $70 \%$ nitric acid can 


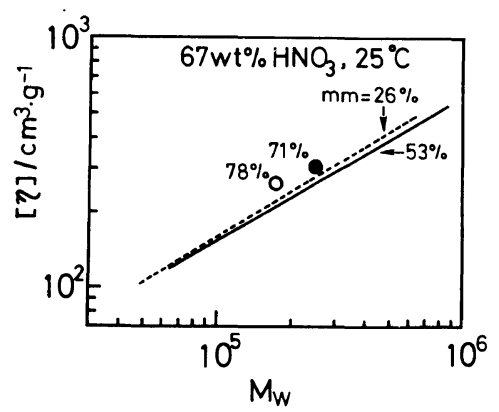

Figure 6. Plots of limiting viscosity number $[\eta]$ of sample code $\gamma-2-2$ (unfilled circle) and $\gamma-2-3$ (filled circle) in $67 \%$ aq. nitric acid soln. against the weight-average molecular weight $M_{w}$ at $25^{\circ} \mathrm{C}$. Solid line, $\gamma$-PAN with $\mathrm{mm}$ (triad tacticity) content of $c a .53 \%{ }^{2}$; broken line, R-PAN in $67 \%$ aq. nitric acid soln. ${ }^{3}$ Numbers on curve or circle mean $\mathrm{mm}$ content $(\%)$.

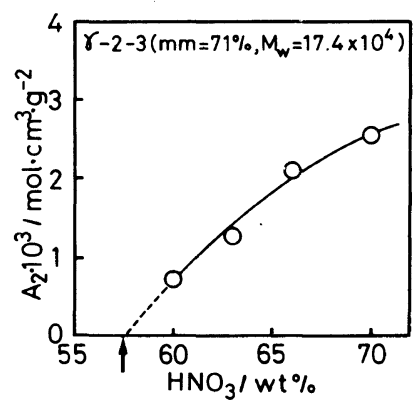

Figure 7. Change in the second virial coefficient $A_{2}$ of a fraction $\gamma-2-3 \quad\left(M_{w}=1.74 \times 10^{5}, m m=71 \%\right)$ with the concentration of nitric acid at $25^{\circ} \mathrm{C}$.

dissolve perfectly-isotactic PAN with $M_{w}$ of $1.5-2.6 \times 10^{5}$ molecularly at room temperature, if it is synthesized.

Figure 6 shows the relationship between $[\eta]$ and $M_{w}$ of the samples $\gamma-2-2$ (unfilled circle) and $\gamma-2-3$ (filled circle) in $67 \%$ aq. nitric acid soln. In the figure the results on the fractions of R-PAN (broken line) ${ }^{3}$ and of $\gamma$-PAN with the tacticity of $\mathrm{ca} .53 \%$ (solid line) ${ }^{2}$ in the same solvent are redrawn from the literature. Apparently, both [ $\eta]$ values of two $\gamma$-PAN fractions with $\mathrm{mm}$ content higher than $70 \%$ have a tendency to be larger than those of other PAN with the same molecular weight, but less isotacticity. Considering the experimental fact that the relation between $[\eta]$ and the number-average molecular weight $M_{n}$ of

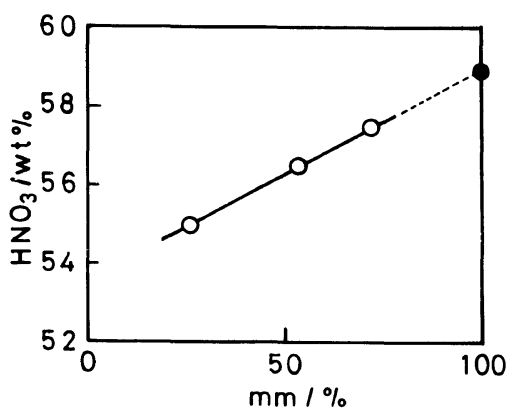

Figure 8. Plots of concentration of nitric acid in aq. nitric acid soln. at the Flory's theta state at $25^{\circ} \mathrm{C}$ versus $\mathrm{mm}$ (triad isotacticity) content of PAN fractions. $\bigcirc$, data points estimated by the second virial coefficient method; , extrapolated point to the triad isotacticity $=100 \%$.

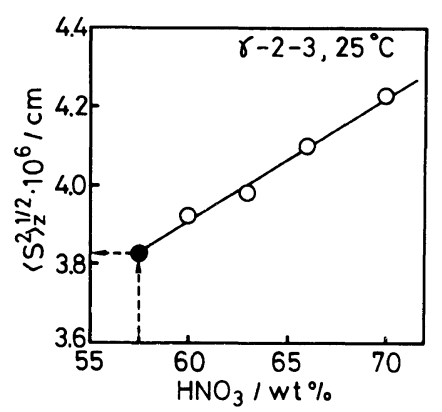

Figure 9. Change in the $z$-average radius of gyration $\left\langle S^{2}\right\rangle_{z}^{1 / 2}$ of a fraction $\gamma-2-3$ in aq. nitric acid soln. at $25^{\circ} \mathrm{C}$ with the nitric acid concentration. $O$, experimental data; O, extrapolated point to a theta solvent.

isotactic polystyrene (PS) in toluene and benzene does not appear to be substantially different from that of atactic PS, ${ }^{10}$ even $70 \%$ aq. nitric acid is not seemed to be as good solvent as toluene and benzene for PS.

Figure 7 shows the dependence of $A_{2}$ of sample code $\gamma-2-3$ on the concentration of nitric acid. Lowering the concentration of nitric acid brings about a linear decrease in $A_{2} . A_{2}$ for PAN with $m m=71.0 \%$ is expected to be zero in $57.5 \%$ aq. nitric acid, which is regarded as a Flory's $\Theta$ solvent, at $25^{\circ} \mathrm{C}$. The concentration of nitric acid in the Flory's $\Theta$ solvent is plotted against $\mathrm{mm}$ content of PAN in Figure 8 , in which the literature data on the fractions with $m m=26 \%$ (sample code R-PAN-4) ${ }^{3}$ and $53 \%$ (sample code $\gamma-1-3)^{2}$ are employed. Evidently 
the nitric acid concentration in Flory's $\Theta$ solvent is higher for PAN with higher regularity. Extrapolation of the solid line to $m m=100 \%$ gives the concentration of nitric acid in the Flory's $\Theta$ solvent for perfectly isotactic PAN to be $c a .59 \%$.

Figure 9 shows $\left\langle S^{2}\right\rangle_{z}^{1 / 2}$ of the sample code $\gamma-2-3$ in aq. nitric acid as a function of the concentration of nitric acid. $\left\langle S^{2}\right\rangle_{z}^{1 / 2}$ shows a marked and monotonical decrease with decreasing the nitric acid concentration as $A_{2}$ does. By extrapolating the line experimentally obtained to the nitric acid concentration at which $A_{2}$ reduces to zero (filled circle), we obtain $\left\langle S^{2}\right\rangle_{z}^{1 / 2}$ at unperturbed state $\left(\left\langle S^{2}\right\rangle_{0, z}^{1 / 2}\right)$ to be $c a .38 .5 \mathrm{~nm}$.

An expansion factor for the radius of gyration $\alpha_{s}$ is defined as the ratio of the weight-average radius of gyration of polymeric chain at perturbed state $\left\langle S^{2}\right\rangle_{w}^{1 / 2}$ and to that at unperturbed state $\left\langle S^{2}\right\rangle_{0, w}^{1 / 2}$ in the form;

$$
\alpha_{\mathrm{s}}=\left\langle S^{2}\right\rangle_{w}^{1 / 2} /\left\langle S^{2}\right\rangle_{0, w}^{1 / 2}
$$

In Figure $10 \alpha_{\mathrm{s}}$ of samples $\gamma-2-3$ (circle), $\gamma-1-3$ $\left(M_{w}=1.68 \times 10^{5} \text { and } m m=53 \%\right)^{2}$ (triangle), and R-PAN-4 $\left(M_{w}=1.58 \times 10^{5}\right)$ (rectangle) in

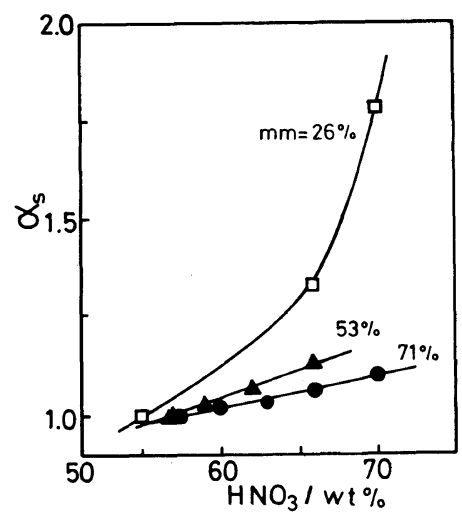

Figure 10. Linear expansion factors for the radius of gyration $\alpha_{\mathrm{s}}$ of fractions $\gamma$-2-3 (circle), R-PAN-4 (triangle), and $\gamma-1-3$ (rectangle) plotted as a function of the concentration of nitric acid. Unfilled mark, calculated value through eq 6 (see the text) using experimentally obtained data on $\left\langle S^{2}\right\rangle_{z}^{1 / 2}$ and $\left\langle S^{2}\right\rangle_{0, z}^{1 / 2}$ and filled mark, calculated value using $\left\langle S^{2}\right\rangle_{z}^{1 / 2}$ experimentally obtained and $\left\langle S^{2}\right\rangle_{0, z}^{1 / 2}$ by the second virial coefficient method. aq. nitric acid soln. plotted aqainst the nitric acid concentration. Here, $\left\langle S^{2}\right\rangle_{w}^{1 / 2}$ and $\left\langle S^{2}\right\rangle_{0, w}^{1 / 2}$ in eq 6 were converted from $\left\langle S^{2}\right\rangle_{z}^{1 / 2}$ and $\left\langle S^{2}\right\rangle_{0, z}^{1 / 2}$, respectively, assuming that PAN fractions obey, regardless of their isotacticity, the Schulz-Zimm type molecular weight distribution function with $M_{w} / M_{n}=1.3^{2} . \alpha_{\mathrm{s}}$ of the samples $\gamma-2-3, \gamma-1-3$ was plotted in the figure as closed mark using $\left\langle S^{2}\right\rangle_{z}^{1 / 2}$ experimentally obtained and $\left\langle S^{2}\right\rangle_{0, z}^{1 / 2}$ which was estimated by extrapolating the relation between $\left\langle S^{2}\right\rangle_{z}^{1 / 2}$ and aq. nitric acid concentration to aq. nitric acid concentration at which $A_{2}=0 . \alpha_{\mathrm{s}}$ of the sample R-PAN-4 (open mark) was calculated using $\left\langle S^{2}\right\rangle_{z}^{1 / 2}$ and $\left\langle S^{2}\right\rangle_{0, z}^{1 / 2}$, which was directly measured in the Flory's $\Theta$ solvent. ${ }^{3}$ Evidently, $\alpha_{s}$ decreases monotonically with a decrease in nitric acid concentration for PAN with a given tacticity and with an increase in stereoregularity of PAN at constant nitric acid concentration, especially in higher concentration region of nitric acid. These remarkable dependence of expansion factor on the stereoregularity of the polymer was also reported on poly(isopropyl acrylate) (PPA) in bromobenzene at $60^{\circ} \mathrm{C}^{11}$ and poly(1-butene) (PB) in toluene at $45^{\circ} \mathrm{C} .{ }^{12}$

Unperturbed chain dimension $A$ is defined by

$$
A \equiv\left(\left\langle R^{2}\right\rangle_{0, w} / M_{w}\right)^{1 / 2}
$$

where $\left\langle R^{2}\right\rangle_{0, w}^{1 / 2}$ is the weight-average end-toend distance at the unperturbed state. Figure 11 represents $A$ of the samples $\gamma-2-3$, R-PAN $-4,{ }^{3}$ and $\gamma-1-3^{2}$ in the Flory's $\Theta$ solvents

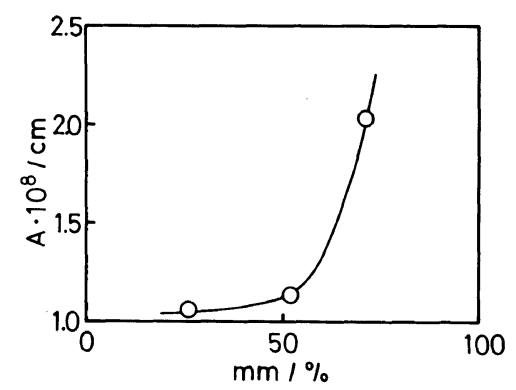

Figure 11. Triad isotacticity $(\mathrm{mm})$ dependence of the unperturbed chain dimension $A$. 


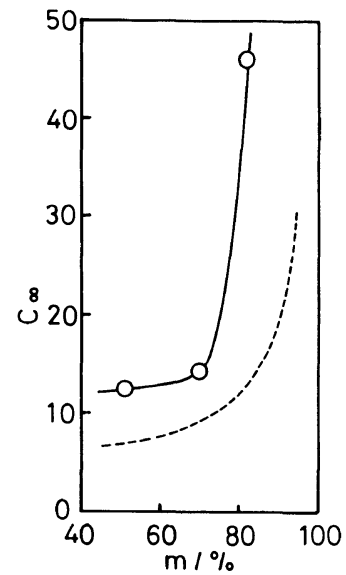

Figure 12. Diad tacticity $(m)$ dependence of characteristic ratio $C_{\infty}$ of PAN. Broken line, Monte Carlo chain with a chain sequence of 100 monomer units. ${ }^{13}$

plotted against the isotactic triad tacticity. Here, $\left\langle R^{2}\right\rangle_{0, w}^{1 / 2}$ in eq 7 was converted to $\left\langle S^{2}\right\rangle_{0, w}^{1 / 2}$ with the assumption that $\gamma$-PAN chain at $\Theta$ state is Gaussian (i.e., $\left\langle R^{2}\right\rangle_{0, w}^{2}=6\left\langle S^{2}\right\rangle_{0, w}^{2}$ ). In aq. nitric acid $A$ of PAN monotonically increases as the tacticity of the polymer increases and the slope of the plot is larger for larger isotacticity.

Using $A$ values we calculated $C_{\infty}$ of PAN chain at the unperturbed state by the following equation:

$$
C_{\infty}=M_{\mathrm{b}} A^{2} / l^{2}
$$

where $M_{\mathrm{b}}$ and $l$ are the mean molecular weight and the length of the skeletal bond, respectively. Figure 12 shows isotactic diad dependence of $C_{\infty}$ for the unperturbed PAN chain (circle). $C_{\infty}$ of various highly isotactic polymers was energetically determined using the data of $A_{2},\left\langle S^{2}\right\rangle_{z}^{1 / 2}$, and $[\eta]$, in particular, from the middle of 1950 s and 1960 s as was briefly summarized by Cowie. ${ }^{1}$ Unfortunately, the tacticity of the polymer samples used had never been determined, because any effective and accurate methods, such as high resolution NMR, had not been available in these days. From late of $1960 \mathrm{~s}^{13,14} C_{\infty}$ has been related to the stereoregularity of the polymers by calculating the conformational energies of meso and racemo diads based on the rotational isomeric state (RIS) model. ${ }^{15}$ Note that inspite of numerous studies on stereoregular polymers no work, in which both $C_{\infty}$ and diad or $\mathrm{mm}$ content have been experimentally determined for given polymer samples, has been carried out. The figure includes also the theoretical curve (Figure 8 in ref. 13) of $C_{\infty} v s$. isotactic diad calculated by Flory et al. ${ }^{13}$ on the basis of RIS model for Monte Carlo chain of polypropyrene with 100 monomer units for each chain. It is obvious that $C_{\infty}$ of PAN noticeably increases, in particular in the range of isotactic diad $>c a .80 \%$ and is always far larger than the calculated value of non-polar Monte Carlo chain over the whole range higher than $50 \%$ isotactic diad. According to Kamide et al.'s analysis ${ }^{4}$ on the ${ }^{13} \mathrm{C}$ NMR spectra of $\gamma$-PAN $(m m=57.6 \%)$ in DMSO- $d_{6}, \gamma$-PAN chain has far longer meso block sequences than those in R-PAN chain and $\mathrm{CN}$ groups in the meso sequence part of $\gamma$-PAN have inherently a double bond nature and may have a transtrans or almost trans-trans conformation. Then, a remarkable or rapid increase in the rigidity of PAN chain with an increase in the stereoregularity (Figure 12) can be explained by the restriction of free rotation due to attractive interaction between adjacent $\mathrm{CN}$ groups along the main chain.

According to the Flory-Fox's expression, ${ }^{16}$ $[\eta]$ is represented by,

$$
[\eta]=\Phi\left(6\left\langle S^{2}\right\rangle_{0}\right)^{3 / 2} \alpha_{s}^{3} / M
$$

where $\Phi$ is Flory's viscosity parameter. For R-PAN $(m m=26 \%)$ and $\gamma$-PAN $(m m=53 \%)$ in $67 \%$ aq. nitric acid solution $\Phi$ is practically independent of the molecular weight, being $2.6 \times 10^{232,3}$ which is in vicinity to theoretical value $\left(2.86 \times 10^{23}\right)$ at perfectly undraining state. ${ }^{17}$ Equation 9 indicates that $[\eta]$ of the solution of a given polymer sample (i.e., with given $M$ ) depends on the two factors, i.e., the radius of gyration at unperturbed state (the chain rigidity) and the expansion factor (the 
excluded volume effect). From the experimental fact that in $67 \%$ aq. nitric acid, $\alpha_{\mathrm{s}}$ monotonically decreases as an increase in the isotacticity (see, Figure 10), it is concluded that maximum value of $[\eta]$ for $\gamma$-PAN with $\mathrm{mm}$ higher than $71 \%$ (Figure 6) is attributed to the highest chain rigidity at unperturbed state.

\section{REFERENCES}

1. See, for example, J. M. Cowie, "Light Scattering from Polymer Solutions," M. B. Huglin, Ed., Academic Press, New York, N.Y., 1972, Chapter 14.

2. K. Kamide, H. Yamazaki, and Y. Miyazaki, Polym. J., 18, 819 (1986).

3. K. Kamide, Y. Miyazaki, and H. Kobayashi, Polym. J., 17, 607 (1985).

4. K. Kamide, H. Yamazaki, K. Okajima, and K. Hikichi, Polym. J., 17, 1291 (1985).

5. See, for example, E. Breitmaier and W. Voelter, "Carbon-13 NMR Spectroscopy," VCH Verlagsgesellschaft, New York, N.Y., VHC, 1987, Chapter 2.

6. E. R. Pike, W. R. M. Pomeroy, and J. M. Vaughan, J. Chem. Phys., 62, 3188 (1975).

7. H. Yamazaki, to be published elsewhere.

8. M. B. Huglin, "Light Scattering from Polymer
Solutions," M. B. Huglin, Ed., Academic Press, New York, N.Y., 1972, Chapter 6.

9. C. Strazille, "Light Scattering from Polymer Solutions," M. B. Huglin, Ed., Academic Press, New York, N.Y., 1972, Chapter 15, p 652.

10. F. Danusso and G. Moraglio, J. Polym. Sci., 24, 161 (1957).

11. W. R. Krigbaum, J. E. Kurz, and P. Smith, J. Phys. Chem., 65, 1984 (1961).

12. J. E. Mark, R. A. Wessling, and R. E. Hughes, $J$. Phys. Chem., 70, 1895 (1966).

13. P. J. Flory, J. E. Mark, and A. Abe, J. Am. Chem. Soc., 88, 639 (1966).

14. See, for example, D. Y. Yoon, U. W. Suter, R. R. Sundararajan, and P. J. Flory, Macromolecules, 8, 784 (1975), E. A. Ojalvo, E. Saiz, R. M. Masegosa, and I. H. Fuentes, Macromolecules, 12, 865 (1979), A. Abe, H. Kobayashi, T. Kawamura, M. Date, T. Uryu, and K. Matsuzaki, Macromolecules, 21, 3414 (1988); R. H. Boyd, and L. Kesner, J. Polym. Sci., Polym. Phys. Ed., 19, 375 (1981); ibid., 19, 393 (1981), L. Carballeira, A. J. Pereiras, and M. A. Risos, Macromolecules, 22, 2668 (1989).

15. P. J. Flory, "Statistical Mechanics of Chain Molecules," Interscience, New York, N.Y., 1969.

16. P. J. Flory and T. G. Fox, J. Am. Chem. Soc., 73, 1904 (1951).

17. P. L. Auer, C. S. Gardner, J. Chem. Phys., 23, 1545 (1955). 